Further Treatment Physical examination revealed tenderness and muscle rigidity in epigastrium. Microperforation was not radiologically confirmed (2x X-Ray, Abdomen CT). PPI, TPN, Nasogastric aspiration and Antibiotics (Co-Amoxiclav changed into Piperacillin/tazobactam because of WBC and CRP elevation) were ordered. Bradycardia after central venous catheter insertion was periodically observed (treated with Theophylline and Atropine). After 2 weeks oral nutrition was gradually included. No changes in duodenal bulb and proper healing pyloric ulcer were discovered in control endoscopy (after 4 weeks).

Discussion

1. Time of PPI Treatment. Our patient was treated for 8 weeks.

2. Surgical treatment was avoided.

3. Possibly early endoscopic intervention might have been considered (no experience).

\section{THE PREVALENCE OF FUNCTIONAL DISEASES OF THE GASTROINTESTINAL TRACT IN CHILDREN OF THE FIRST YEAR OF LIFE LIVING IN THE BELGOROD REGION (RUSSIAN FEDERATION)}

M Gurova Margarita*, I Kirienko Arina, A Procenko Ekaterina, A Sviridov Alexandr, A Romanova Tatiana, V Podsvirova Elena, S Popova Valentina. St. Petersburg State Pediatric Medical University

\subsection{6/archdischild-2021-europaediatrics.235}

Goal To study the prevalence of functional disorders of the gastrointestinal tract in children of the first year of life living in the Belgorod region, according to the questionnaire data.

Methods We conducted a survey by questioning 348 women with children aged 1 year to 4 years. All children were born full-term. The ratio of boys to girls was 1: $1(47.7 \% / 166$ boys and 52.3\%/ 182 girls).

Results According to the questionnaire, gastroenterological complaints were found in $82.8 \% / 288$ children. Most often among functional disorders were detected regurgitation syndrome $(18.3 \% / 64)$, infantile colic $(74,4 \% / 259)$ and constipation $(33 \% / 115)$. In $25.9 \%$ cases children had two or more functional disorders, more commonly infantile colic plus constipation.

Among the factors predisposing to the development of functional disorders of the gastrointestinal tract, the following were identified: aggravated pregnancy (31.3\%) and childbirth (operative delivery - 25.3\%), features of the feeding (early termination of breastfeeding up to 3 months of life in $29.8 \%$ ), antibiotic therapy in the first months of life $-23.6 \%$.

For medical attention/treatment applied $56.25 \%$ parents of children with regurgitation, 67,2\% parents of children with infantile colic and $57,4 \%$ with constipation.

The use of diet in treatment of functional disorders was most effective in patients with regurgitation syndrome $(57 \%)$ and constipation (41.7\%), while in the case of intestinal colic - only in $31.7 \%$ of cases.

Conclusion Based on a questionnaire survey of 384 parents of children from

1 year to 4 years, living in the Belgorod region, we found a high frequency of detecting of gastroenterological complaints - in $82.8 \%$ of cases. Among the functional diseases of the gastrointestinal tract, the most common were regurgitation syndrome $(18.3 \%)$, infantile colic $(74.4 \%)$, and functional constipation (33\%). Most often sought medical help parents of children with infantile colic. The diet was effective in most cases of functional gastrointestinal disorders except in cases of infantile colic

\section{NEW NAME, NEW CRITERIA, OLD DISEASE: PAEDIATRIC INTESTINAL PSEUDOOBSTRUCTION (PIPO)}

Ana Mocic Pavic*, Zrinjka Mišak, Ivana Trivić, Marko Mesić, Kristina Baraba Dekanić, Sanja Kolaček, Iva Hojsak. Children's hospital Zagreb

\subsection{6/archdischild-2021-europaediatrics.236}

Introduction Pediatric intestinal pseudoobstruction (PIPO) is a rare and complex gastrointestinal motility disorder affecting mainly the small intestine and the colon resulting in intestinal failure.

Due to severe and reccurent episodes of pseudo-obstruction, children are unable to meet their nutritional needs enterally and might even need parental nutritional support. In 2018, diagnostic criteria were proposed by the European Society for Pediatric Gastroenterology, Hepatology and Nutrition with a goal to improve diagnosis, management and treatment while decreasing morbidity and mortality in these patients.

Case Report We present a case of female infant who was referred to the tertiary center for further medical evaluation due to recurrent abdominal distention and subileus at the age of 6 months. Prenatally, the child was diagnosed with megacystis, later evaluated as hypotonic urinary bladder without vesicouretheral reflux and with normal renal function. At four months, she experienced the first intestinal obstruction characterized with severe abdominal distention, stool absence and inability to tolerate enteral feeds. Mechanical obstruction was excluded by radiologic studies. Therefore she was transferred to our center for further diagnostic work-up. In accordance to the guidelines, in our center, rectal biopsy was performed to exclude Hirschsprung's disease. During initial evaluation, the patient experienced a new episode of distention and not tolerating oral feeds.

Radiologic images during the episode showed distended bowel loops throughout the abdomen (Figure 1). She was treated by withholding enteral feed, intravenous fluids and prokinetics. Because of the progressive course of the distension she underwent exploratory laparotomy with formation of decompressive ileostomy at the age of 7 months (Figure 2). Full thickness biopsy were also performed at various site of small and large intestine and showed histological elements of intestinal neuronal dysplasia.

In her further clinical course she experienced 3 more episodes treated conservatively, she was not able to tolerate full enteral feeds so we initiated parenteral nutrition to maintain adequate growth.

In accordance with disease course so far, our patient met 2 out of 4 listed criteria for pediatric pseudointestinal obstruction, persistently dilated loops of small intestine with air fluid and inability to maintain adequate nutrition and growth via enteral feed.

Conclusion To conclude, we present a difficult case of PIPO, with early onset of abdominal distention, need for parenteral nutrition, and bladder involvement with uncertain prognosis.

Nevertheless, early recognition of disorder leads to optimal management including formation of venting stoma, prevention of unnecessary repeated surgeries and better nutritional management. 